\title{
Development of a Highly Reproducible System to Evaluate Inhibition of Cytochrome P450 3A4 Activity by Natural Medicines
}

\author{
Yu Sato, Takamitsu Sasaki, Shogo Takahashi, Takeshi Kumagai, Kiyoshi Nagata \\ Department of Environmental and Health Science, Tohoku Pharmaceutical University, Miyagi, Japan.
}

Received, June 2, 2015; Revised, July 17, 2015; Accepted, July 28, 2015; Published, August 4, 2015.

\begin{abstract}
PURPOSE: In recent years, a number of natural medicines have been reported to have inductive or inhibitive effects on the activity of drug metabolizing enzymes, upon co-administration with prescribed medicines. However, information regarding natural medicine-drug interactions that influence drug metabolism is limited owing to the lack of efficient screening method for such interactions. Therefore, to understand whether P450 activity is affected by natural medicine, we have established frozen recombinant P450-expressing cells infected with human CYP3A4 expressing adenovirus (Ad-CYP3A4) to evaluate the effect of natural medicines on CYP3A4 activity. METHODS: Ad-CYP3A4 cells were created by infecting HepG2 cells with Ad-CYP3A4 at 10 multiplicity of infection (MOI) and these cells were stored using cryopreservation medium (fAd-CYP3A4 cells) to obtain long-term consistent data and stable supplies of cells expressing a constant level of CYP3A4 activity. RESULTS: The CYP3A4 activity in fAd-CYP3A4 cells remained unaffected at the end of each frozen period $(0,1,2$, and 6 months). Inhibitory effect on CYP3A4 activity by typical inhibitors (ketoconazole, hyperforin) and natural medicines (Cat's Claw, Devil's Claw, Feverfew, Peppermint Oil, Red Clover, and Siberian Eleuthero) were evaluated. The inhibitors had nearly equal $\mathrm{IC}_{50}$ values in fAd-CYP3A4 cells, Ad-CYP3A4 cells and recombinant CYP3A4 microsomes. Cat's Claw, Peppermint Oil and Siberian Eleuthero inhibited CYP3A4 activity more potently than $0.1 \mu \mathrm{M}$ ketoconazole in fAd-CYP3A4 cells. CONCLUSIONS: In the present study, we have successfully developed a highly reproducible system to evaluate CYP3A4 inhibition by natural medicines.
\end{abstract}

This article is open to POST-PUBLICATION REVIEW. Registered readers (see "For Readers") may comment by clicking on ABSTRACT on the issue's contents page.

\section{INTRODUCTION}

Cytochromes P450 (P450) belong to a supergene family of monooxygenases and play an important role in the oxidative metabolism of endogenous compounds such as steroids, fatty acids, and prostaglandins and exogenous compounds such as drugs, environmental pollutants, and food components $(1,2)$. Of all the CYPs, CYP3A4, CYP2D6, CYP2C19, CYP2C9, and CYP1A2 are mainly expressed in human livers and are involved in $\sim 90 \%$ of the oxidative metabolism of drugs (1). In particular, CYP3A4 is the most abundant CYP form found in livers and small intestines, comprising approximately $30 \%$ and $70 \%$ of the total P450 content, respectively (3). CYP3A metabolizes more than $50 \%$ of the currently used therapeutic drugs, for example, alprazolam, and nifedipine $(3,4)$. Thus, compounds that inhibit the CYP3A4 activity could potentially affect the pharmacokinetics/pharmacodynamics of these therapeutic drugs. For example, grapefruit juice (GFJ) has been found to increase the bioavailability of felodipine (5-7). GFJ reduces the first-pass effect of felodipine by inhibition of its metabolism by CYP3A4. Therefore, it is important to evaluate not only drug-drug interaction but also drug-dietary substance or drug-natural product interaction.

Natural medicines have been used for various health conditions worldwide. These have been used for self-medication without medical supervision and are believed to be safe. In recent years, a number of natural medicines have been reported to have inductive or inhibitive effects on the activity of drug metabolizing enzymes, upon co-administration with prescribed medicines. A typical example is St. John's wort (SJW), which increases the activity of CYP3A4 and reduces plasma concentrations of a number of drugs (7-9). However, the information regarding natural medicine-drug interactions that influence drug

\footnotetext{
Corresponding Author: Kiyoshi Nagata; Department of Environmental and Health Science, Tohoku Pharmaceutical University; 4-4-1 Komatsushima, Aoba-ku, Sendai, Miyagi, Japan; E-mail: nagataki@tohoku-pharm.ac.jp
} 
metabolism is limited. Therefore, one can assume that natural medicine-drug interactions pose an important problem for clinical therapeutics and it is essential to understand whether P450 activity is affected by natural medicine.

At present, human liver microsomes and primary human hepatocytes are the most desirable enzyme sources for drug metabolism experiments since they retain majority of the biological functions of human livers as well abundantly express drug-metabolizing enzymes (10-12). However, these show large donor-to-donor variability in drug metabolizing activity, which make date interpretation from different sources difficult (10-12). Human hepatoma-derived cell lines such as HepG2 cells have been used for in vitro drug metabolism studies owing to have an unlimited lifespan, but have drawback the limit of liver-specific functions including expression of drug-metabolizing enzymes (13-15). In order to compensate for the shortcomings of HepG2 cells, various transient $\mathrm{P} 450$ expression systems are generally used for drug metabolism studies.

On the other hand, viral and non-viral methods have been used as tools for intracellular gene transfer (16). In particular, adenoviral vectors are capable of introducing target genes into a variety of cells efficiently (17). The expression level of a recombinant protein can be easily controlled by transfection, depending on the amount of adenovirus. However, this process requires infection of adenovirus with biosafety regulation before each experiment and, a well-to-well difference in enzyme activity is observed within the same experiment, thus, making this system unsuitable for high-throughput screening. In order to overcome these problems, we have established frozen recombinant $\mathrm{P} 450$-expressing cells infected with human CYP3A4 expressing adenovirus (Ad-CYP3A4) to evaluate the effect of natural medicines on CYP3A4 activity. By create large amounts of the cells simultaneously, it is possible to obtain long-term consistent data and stable supplies of cells expressing a constant level of P450 activity.

In the present study, we examined the difference in enzymatic activity between the frozen Ad-CYP3A4-infected cells (fAd-CYP3A4 cells) and non-frozen Ad-CYP3A4-infected cells (Ad-CYP3A4 cells), and by using our new method based on fAd-CYP3A4 cells, we evaluated the extent of CYP3A4 inhibition by natural medicines.

\section{METHODS}

\section{Materials}

Ketoconazole was obtained from LKT Laboratories (St. Paul, MN). Hyperforin was obtained from Sigma-Aldrich (St. Louis, MO). Dimethyl sulfoxide (DMSO) was obtained from Nacalai Tesque (Kyoto, Japan). Oligonucleotides were commercially synthesized by Fasmac (Atsugi, Japan). Microsomes expressing human CYP3A4 prepared from baculovirus-infected insect cells (recombinant CYP3A4 microsomes) were purchased from BD Gentest (Woburn, MA). Cat's Claw, Devil's Claw, Feverfew, Peppermint Oil, Red Clover, and Siberian Eleuthero were obtained from Nature's way (Springville, UT).

\section{Cell Culture}

HepG2 and HEK293 cells were obtained from Riken cell bank (Tsukuba, Japan). Cells were cultured in Dulbecco's modified Eagle's medium (DMEM; WAKO Pure Chemicals, Osaka, Japan) supplemented with $10 \%$ fetal bovine serum (FBS; Biowest, Miami, FL), minimum essential medium nonessential amino acids (MEM NEAA; Invitrogen, Carlsbad, CA), and Antibiotic-Antimycotic (Invitrogen), under $5 \% \mathrm{CO}_{2}$ at $37^{\circ} \mathrm{C}$. Before $24 \mathrm{~h}$ adenovirus infection, HepG2 cells were seeded at $1.5 \times 10^{5}$ cells per well onto 24 -well tissue culture plates (BD Biosciences, Heidelberg, Germany) for real-time PCR, $1.0 \times 10^{5}$ cells per well onto 48 -well tissue culture plates (BD Biosciences) for immunoblotting, and $0.5 \times 10^{5}$ cells per well onto 96-well tissue culture plates (BD Biosciences) for P450-Glo ${ }^{\mathrm{TM}}$ assay. The cells were subjected to corresponding experiments after $48 \mathrm{~h}$.

\section{Sample Preparation}

Ketoconazole and hyperforin were dissolved in DMSO and the final assay concentration ranges were $0.01-10 \mu \mathrm{M}$ (ketoconazole) and 0.1-100 $\mu \mathrm{M}$ (hyperforin). For the assessment of inhibitory activities, the daily dose of the natural medicines was extracted in $10 \mathrm{~mL}$ ethanol $70 \%(\mathrm{v} / \mathrm{v})$ for $2 \mathrm{~h}$ in water-bath at $37^{\circ} \mathrm{C}$. The resulting solution was centrifuged at $3,500 \mathrm{rpm}$ for $15 \mathrm{~min}$ at $4{ }^{\circ} \mathrm{C}$ and the supernatants were used as natural medicine extracts. These were added to the culture medium $(0.1 \%$, $0.25 \%$, and $1 \%$ extracts) for CYP3A4 activity assay.

\section{Construction of Recombinant Adenovirus}

The open reading frame of CYP3A4 DNA was isolated by amplification of cDNA obtained from 
human hepatocyte mRNA by PCR using the forward primer, 5'-CACCATGGCTCTCATCCCAGACTTGGC-3', and the reverse primer, 5'-TCAGGCTCCACTTACGGTGCCATC-3'. The synthesis was carried out according to the manufacturer's protocol for the ViraPower ${ }^{\mathrm{TM}}$ Adenoviral Expression System, pENTR $^{\mathrm{TM}}$ Directional TOPO Cloning and pAd/CMV/V5-DEST ${ }^{\text {TM }}$ Gateway ${ }^{\circledR}$ Vector kits (Invitrogen). The plasmid was purified by $\mathrm{CsCl}$ density gradient centrifugation, linearized with PacI (New England Biolabs Inc, Ipswich, MA) and then transfected into HEK293 cells using Targefect F-1 (Nacalai Tesque). The control adenovirus, a $\beta$-galactosidase-expressing adenovirus (Ad-LacZ) was produced using the $\mathrm{pAd} / \mathrm{CMV} / \mathrm{V} 5-\mathrm{GW} / \mathrm{lacZ}$ control plasmid (Invitrogen). The titer of adenovirus, $50 \%$ titer culture infectious dose ( TCID $_{50}$ ), was determined in HEK293 cells. TCID 50 has been reported almost equivalent to that of the plaque-forming unit (18). Multiplicity of infection (MOI) was calculated by dividing TCID $_{50}$ by the number of cells at the time of seeding.

\section{Preparation of Frozen Ad-CYP3A4 Infected HepG2 Cells}

HepG2 cells were seeded at $3 \times 10^{6}$ cells in $100-\mathrm{mm}^{2}$ culture dish (BD Biosciences) $24 \mathrm{~h}$ before Ad-CYP3A4 infection at $10 \mathrm{MOI}$. After $24 \mathrm{~h}$, the cells were harvested, media was replaced with cryopreservation medium (Bambanker; Nippon Genetics, Tokyo), and cells were stored in liquid nitrogen. fAd-CYP3A4 cells were thawed in a $37^{\circ} \mathrm{C}$ water-bath and then washed with cultured medium. The cells were seeded into $60-\mathrm{mm}^{2}$ culture dish (BD Biosciences) and incubated in fresh medium for $24 \mathrm{~h}$. The cells were seeded at $1.5 \times 10^{5}$ cells per well onto 24-well tissue culture plates for real-time PCR, $1.0 \times 10^{5}$ cells per well onto 48 -well tissue culture plates for immunoblotting, and $0.5 \times$ $10^{5}$ cells per well onto 96 -well tissue culture plates for $\mathrm{P} 450-\mathrm{Glo}^{\mathrm{TM}}$ assay, respectively, for $24 \mathrm{~h}$ prior to experiments.

\section{Quantitative Analysis of CYP3A4 mRNA Contents}

Total RNA was extracted by using TRI

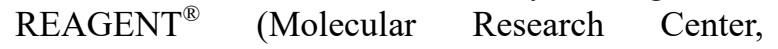
Cincinnati, $\mathrm{OH})$. First-strand cDNA was synthesized from $2 \mu \mathrm{g}$ total RNA in a $20 \mu \mathrm{L}$ reaction mixture using Moloney Murein Virus Reverse Transcriptase (Promega, Madison, MI), oligo $(\mathrm{dT})_{20}$ primer, and Ribonuclease Inhibitor
(TaKaRa Bio, Shiga, Japan). The cDNA was used to carry out real-time polymerase chain reaction (PCR) using SYBR ${ }^{\circledR}$ Premix ExTaq (TaKaRa Bio) in order to measure mRNA levels of CYP3A4 and glyceraldehyde-3-phosphate dehydrogenase $(G A P D H)$. The amplification reactions were performed with specific primers for CYP3A4 (forward:

5'-CTGTGTTTCCAAGAGAAGTTAC-3' and reverse: TGCATCAATTTCCTCCTGCAG-3'), and GAPDH (forward: 5'-CATGGGTGTGAACCATGAGAA-3' and reverse: 5'-GGTCATGAGTCCTTCCACGAT-3') (19). Quantitative values were obtained above the threshold PCR cycle number $(\mathrm{Ct})$ at which the increase in signal associated with an exponential growth in PCR products was detected using Thermal Cycler Dice ${ }^{\mathrm{TM}}$ TP800 (TaKaRa Bio). The relative mRNA expression levels in each sample were normalized to those of GAPDH.

\section{Determination of CYP3A4 Enzymatic Activities using P450-Glo ${ }^{\mathrm{TM}}$ Assay}

CYP3A4 activity was measured using a P450-Glo ${ }^{\text {TM }}$ CYP3A4 assay kit (Promega) according to the manufacturer's protocol. Ad-CYP3A4 and fAd-CYP3A4 cells were rinsed twice with phosphate buffer saline. Fresh medium containing $3 \mu \mathrm{M}$ luciferin-IPA and/or test compound was added to the cells and incubated at $37^{\circ} \mathrm{C}$ for $1 \mathrm{~h}$. Subsequently, $40 \mu \mathrm{L}$ of the medium from each well was transferred to a 96-well white luminometer plate (BD Biosciences), and the luminescence was detected by using a Glomax ${ }^{\mathrm{TM}}$ 96 microplate luminometer (Promega). The luminescence signals were calculated by subtracting background luminescence values (no-cell control) from the values of the test compound and the blank (without the test compound).

The reaction mixture consisted of recombinant CYP3A4 microsomes, potassium phosphate buffer, luciferin-IPA (final concentration is $2 \mathrm{nM}, 100 \mathrm{mM}$ and $3 \mu \mathrm{M}$, respectively) and/or test compounds in a final volume of $25 \mu \mathrm{L}$. The mixture was incubated at $37^{\circ} \mathrm{C}$ for $20 \mathrm{~min}$. At the end of the reaction period, reaction mixture was transferred to a 96-well white luminometer plate, and the CYP3A4 activity was measured as described above.

\section{Immunoblot Analysis}

Immunoblot analysis was performed to detect the expression of CYP3A4 proteins in $\mathrm{HepG} 2$, Ad-CYP3A4, and fAd-CYP3A4 cells. Whole cell 
lysates from these cells were size-fractionated by gel electrophoresis on a $10 \%$ polyacrylamide $/ 0.1 \%$ sodium dodecyl sulfate gel after being denatured by heating in a 2-mercaptoethanol-containing loading buffer. The proteins were electrotransferred to Immobilon $^{\circledR}-\mathrm{P}$ membranes (Millipore, Billerica, $\mathrm{MA}$ ), which were then incubated for $1 \mathrm{~h}$ with polyclonal goat anti-human CYP3A4 antibody (Santa Cruz Biotechnology, Inc., Santa Cruz, CA) that had been diluted in Tris-buffered saline $(1: 1,000)$. The membranes were subsequently incubated for $1 \mathrm{~h}$ with a horseradish peroxidase-conjugated secondary antibody diluted in Tris-buffered saline $(1: 10,000$; Santa Cruz Biotechnology, Inc.). After development with SuperSignal West Pico chemiluminescent substrate (Thermo Fisher Scientific Inc., Waltham, MA), the membrane was scanned using LAS-4000 (GE Healthcare, Milwaukee, WI).

\section{STATISTICAL ANALYSIS}

Data are expressed as the mean \pm standard (S.D.) error from three determinations. Statistical comparison of two groups was performed using a two-tailed Student's $t$-test. $P$-values less than 0.05 were considered to be statistically significant.

\section{RESULTS}

Determination of optimal conditions for CYP3A4 expression in Ad-CYP3A4-infected HepG2 cells

To optimize the conditions of CYP3A4 expression in Ad-CYP3A4 cells, HepG2 cells were infected with various amounts $(0,3,5,10$, and $20 \mathrm{MOI})$ of Ad-CYP3A4 and measured mRNA expression, enzyme activity, and protein expression. The levels of CYP3A4 mRNA expression, activity, and protein increased corresponding to the infection amount of Ad-CYP3A4, but not in Ad-LacZ infected cells at 20 MOI (Figure 1). The highest activity was measured in cells infected with Ad-CYP3A4 at 20 MOI, however this infection level was found to be cytotoxic. Therefore, we used Ad-CYP3A4 at 10 MOI in order to prepare the Ad-CYP3A4 cells.

\section{Effects of cryopreservation on CYP3A4 expression}

fAd-CYP3A4 cells were prepared by infecting HepG2 cells with Ad-CYP3A4 at $10 \mathrm{MOI}$ and these cells were stored using cryopreservation medium. To confirm the effects of cryopreservation on
CYP3A4 expression in fAd-CYP3A4 cells, we compared CYP3A4 mRNA, activity and protein expression between fAd-CYP3A4 cells and non-frozen Ad-CYP3A4 cells at 10 MOI. The mRNA, activity, and protein expression levels of CYP3A4 were upregulated in fAd-CYP3A4 cells, as compared to the HepG2 cells (Figure 2). In fAd-CYP3A4 cells, these levels showed higher values as compared to the Ad-CYP3A4 cells. Moreover, to confirm the effects of cryopreservation period on CYP3A4 activity in fAd-CYP3A4 cells, we measured the activity after cryopreservation at the end of $0,1,2$, and 6 months. As the results, CYP3A4 activity remained unaffected at the end of each frozen period (Figure 3). In those periods, the activities were not significantly different.

Effects of specific inhibitors on CYP3A4 activity In order to demonstrate the utility of fAd-CYP3A4 cells for the evaluation of inhibition of CYP3A4 enzyme activity, we used ketoconazole and hyperforin as typical inhibitors (20). When fAd-CYP3A4 cells, Ad-CYP3A4 cells, and recombinant CYP3A4 microsomes were treated with ketoconazole or hyperforin, enzyme activity of CYP3A4 was decreased in a concentration-dependent manner (Figure 4). $\mathrm{IC}_{50}$ values of $0.077,0.105$, and $0.096 \mu \mathrm{M}$ for ketoconazole were determined in fAd-CYP3A4 cells, Ad-CYP3A4 cells, and recombinant CYP3A4 microsomes, respectively. Hyperforin, a compound of SJW, were inhibited CYP3A4 activity at $\mathrm{IC}_{50}$ values of $9.212,6.738$, and $4.411 \mu \mathrm{M}$, respectively (Table 1). The $\mathrm{IC}_{50}$ values of ketoconazole and hyperforin showed nearly equal values in each evaluation systems.

Effects of natural medicines on CYP3A4 activity In order to substantiate the ability of fAd-CYP3A4 cells to assess CYP3A4 activity inhibition by natural medicines, the cells were treated with natural medicine extracts. As shown in Figure 5, each natural medicine extract inhibited the activity in a concentration-dependent manner. $1 \%$ extracts of Cat's Claw, Peppermint Oil, and Siberian Eleuthero strongly inhibited CYP3A4 activity in fAd-CYP3A4 cells (Figure 5A). The inhibitory levels of Devil's Claw, Feverfew, and Red Clover were found to be comparable to $0.1 \mu \mathrm{M}$ ketoconazole (positive control) in fAd-CYP3A4 cells. 

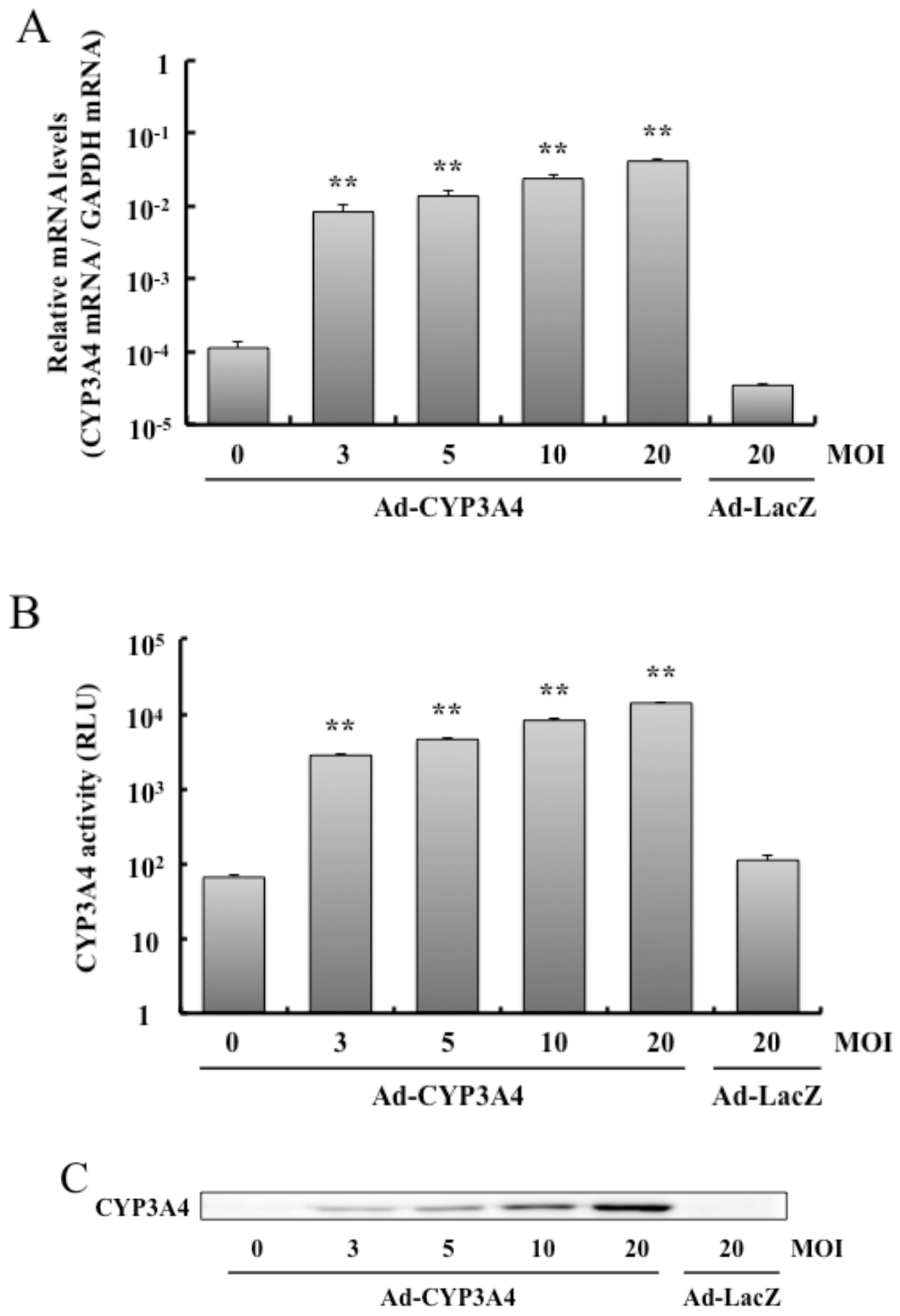

Figure 1. MOI-dependent increase of CYP3A4 levels in HepG2 cells infected with Ad-CYP3A4.

HepG2 cells were seeded in 24-well tissue culture plates at $1.5 \times 10^{5}$ cells per well for real-time PCR, 96-well tissue culture plates at $0.5 \times 10^{5}$ cells per well for P450-Glo ${ }^{\mathrm{TM}}$ assay, and 48 -well tissue culture plates at $1.0 \times 10^{5}$ cells per well for immunoblotting. The next day, the cells were infected with Ad-CYP3A4 (MOI of 3, 5, 10, and 20) or Ad-LacZ (MOI of 20). After 48 h, mRNA expression (A), enzyme activity (B), and protein levels (C) were measured using real-time PCR, P450-Glo ${ }^{\mathrm{TM}}$ assay, and immunoblotting. Results represent the mean \pm S.D. $(\mathrm{n}=3)$. Student's $t$-test: $* * p<0.01$, compared with non-infection cells (0 MOI). 

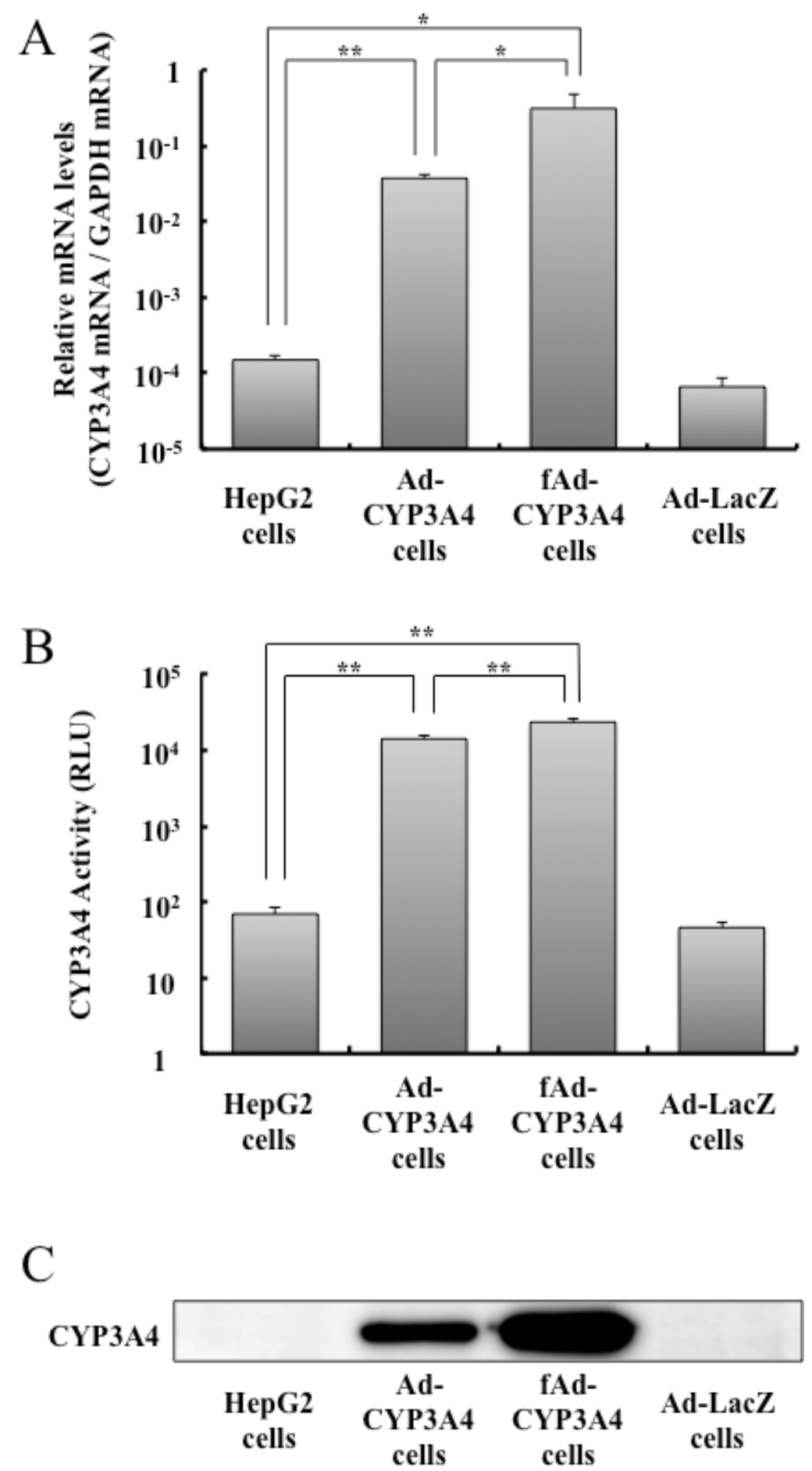

Figure 2. Comparison of CYP3A4 levels in fAd-CYP3A4 cells and Ad-CYP3A4 cells.

HepG2 cells were seeded in 24-well tissue culture plates at $1.5 \times 10^{5}$ cells per well for real-time PCR, 96-well tissue culture plates at $0.5 \times 10^{5}$ cells per well for P450-Glo ${ }^{\mathrm{TM}}$ assay, and 48 -well tissue culture plates at $1.0 \times 10^{5}$ cells per well for immunoblotting. The next day, the cells were infected with Ad-CYP3A4 (MOI of 10) or Ad-LacZ (MOI of 10). After 48 h, mRNA expression (A), enzyme activity (B), and protein levels (C) were measured using real-time PCR, P450-Glo ${ }^{\mathrm{TM}}$ assay, and immunoblotting. fAd-CYP3A4 cells were seeded in the same way as HepG2 cells before $24 \mathrm{~h}$ of real-time PCR, P450-Glo ${ }^{\mathrm{TM}}$ assay, and immunoblotting. Results represent the mean \pm S.D. $(\mathrm{n}=3$ ). Student's $t$-test: $* * p<0.01, * p<0.05$. 


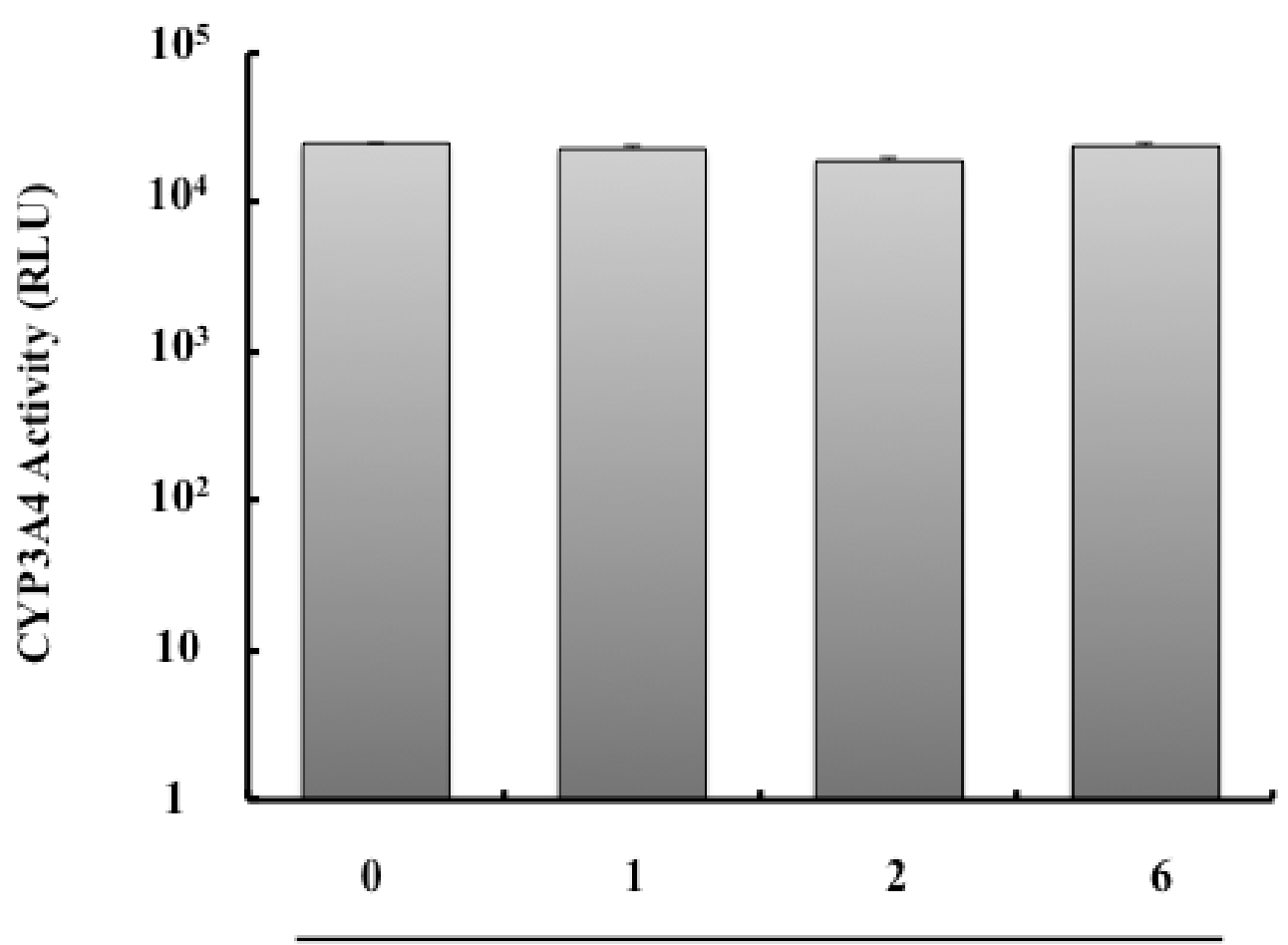

\section{After cryopreservation (month)}

Figure 3. Effect of the frozen period on CYP3A4 activity.

After cryopreservation for $0,1,2$ and 6 months, fAd-CYP3A4 cells were seeded in 96-well tissue culture plates at $0.5 \times$ $10^{5}$ cells per well. After $24 \mathrm{~h}$, enzyme activity was measured using P450-Glo ${ }^{\mathrm{TM}}$ assay. Results represent the mean \pm S.D. $(\mathrm{n}=3)$.

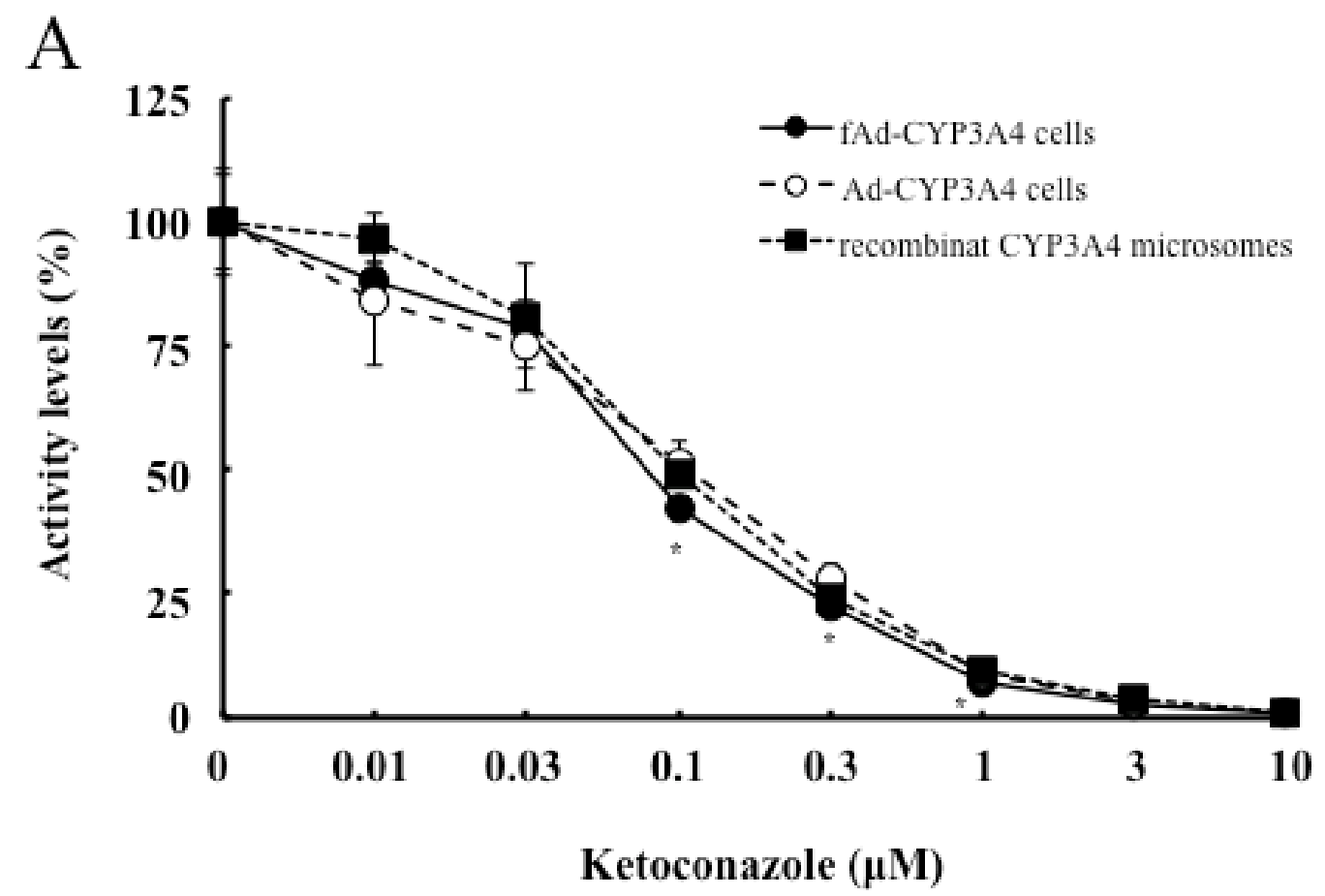

Figure 4. 


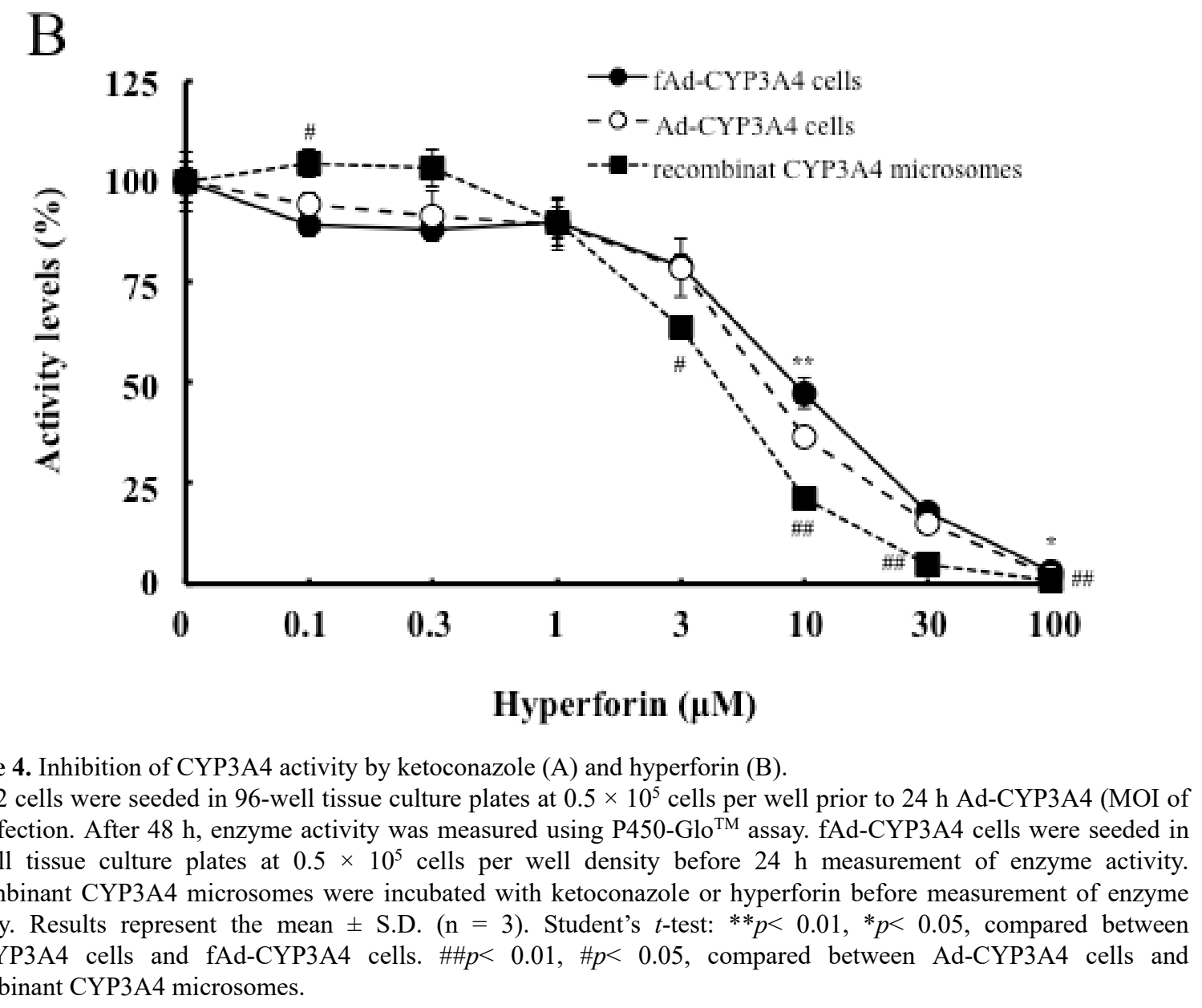

Figure 4. Inhibition of CYP3A4 activity by ketoconazole (A) and hyperforin (B).

HepG2 cells were seeded in 96-well tissue culture plates at $0.5 \times 10^{5}$ cells per well prior to $24 \mathrm{~h}$ Ad-CYP3A4 (MOI of 20 ) infection. After $48 \mathrm{~h}$, enzyme activity was measured using P450-Glo ${ }^{\mathrm{TM}}$ assay. fAd-CYP3A4 cells were seeded in 96-well tissue culture plates at $0.5 \times 10^{5}$ cells per well density before $24 \mathrm{~h}$ measurement of enzyme activity. Recombinant CYP3A4 microsomes were incubated with ketoconazole or hyperforin before measurement of enzyme activity. Results represent the mean \pm S.D. $(\mathrm{n}=3)$. Student's $t$-test: ${ }^{*} p p<0.01,{ }^{*} p<0.05$, compared between Ad-CYP3A4 cells and fAd-CYP3A4 cells. $\# \#<<0.01, \# p<0.05$, compared between Ad-CYP3A4 cells and recombinant CYP3A4 microsomes.

Table 1. $\mathrm{IC}_{50}$ values of CYP3A4 activity using each evaluation systems

\section{Evaluation systems}

\section{$\mathrm{IC}_{50}(\mu \mathrm{M})$}

Ketoconazole

Hyperforin

fAd-CYP3A4 cells $\quad 0.077(0.075-0.079) \quad 9.212(8.19-10.234)$

Ad-CYP3A4 cells $\quad 0.105(0.084-0.126) \quad 6.738(6.214-7.262)$

Recombinat CYP3A4 microsomes

$$
0.096(0.091-0.101) \quad 4.411(4.158-4.664)
$$

$\underline{\mathrm{IC}_{50} \text { values were determined with data shown in Figure } 4 .}$ 

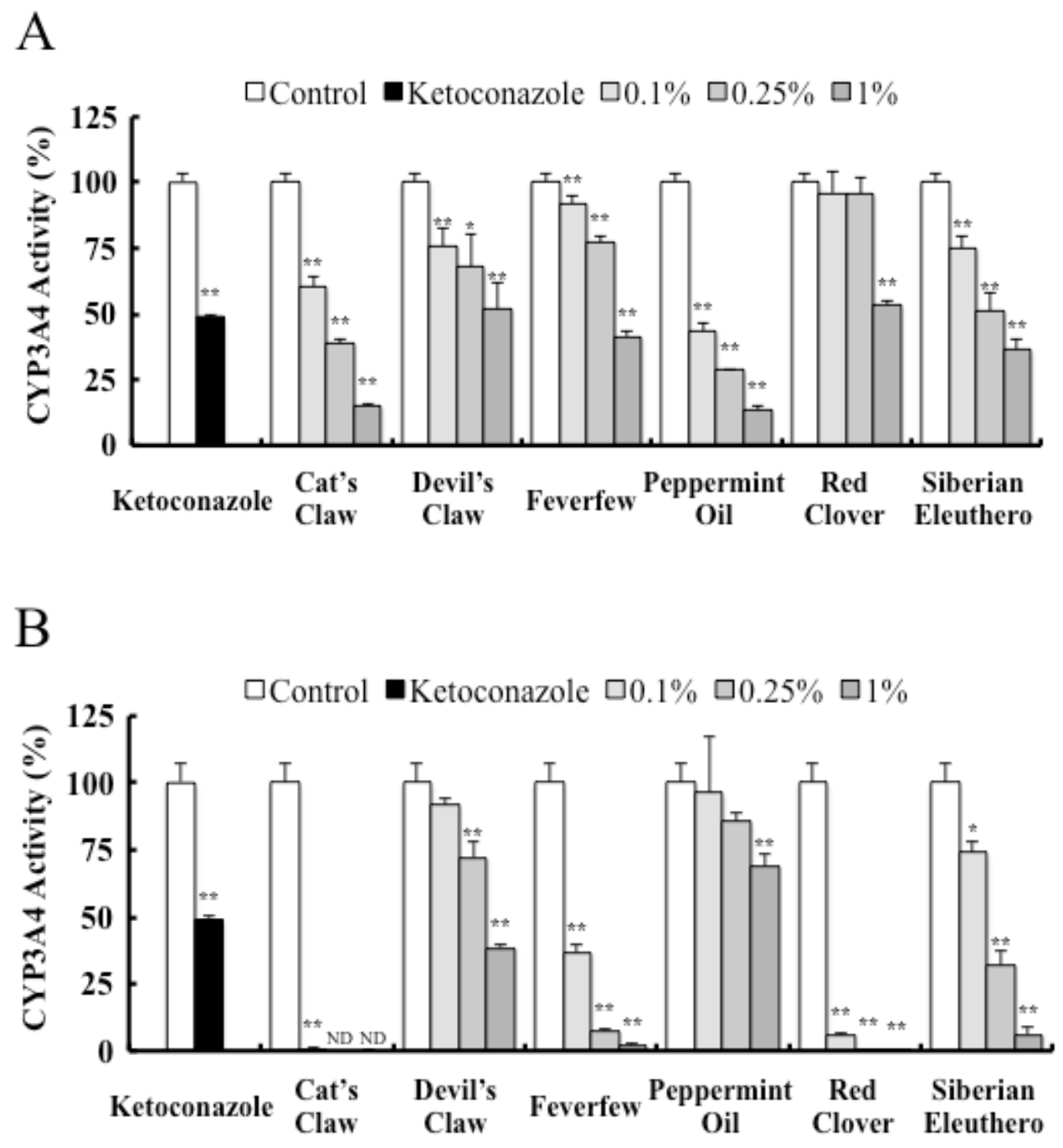

Figure 5. Inhibitory effect of natural medicines on CYP3A4 activity in fAd-CYP3A4 cells (A) and CYP3A4 microsomes (B).

fAd-CYP3A4 cells were seeded in 96-well tissue culture plates at $0.5 \times 10^{5}$ cells per well. After 24 h, enzyme activity of CYP3A4 was measured. Reaction mixture of recombinant CYP3A4 microsomes was incubated with ketoconazole $(0.1 \mu \mathrm{M})$ and test natural medicine compounds. The enzyme activity was measured using P450-Glo ${ }^{\mathrm{TM}}$ assay. The values of enzyme activity in untreated groups were set equal to $100 \%$. Results represent the mean \pm S.D. $(n=3)$ ND, not detected. Student's $t$-test: $* * p<0.01, * P<0.05$, compared with control.

On the other hand, in recombinant CYP3A4 microsomes, CYP3A4 activity was strongly inhibited by $1 \%$ extracts of Cat's Claw, Feverfew, Red Clover, and Siberian Eleuthero, which was stronger than that of the finding in fAd-CYP3A4 cells (Figure 5B). However, this was not observed in case of Peppermint Oil.

\section{DISCUSSION}

Adenoviral vectors are highly efficient tools for gene transfer into various cell types. Furthermore, expression levels of the target protein can be controlled by the infection amount of the adenovirus. As shown in Figure 1, Ad-CYP3A4 infection leads to increased CYP3A4 mRNA, protein levels, and enzyme activity in HepG2 cells and this increase corresponds to the infection amount of Ad-CYP3A4. However, it may cause a well-to-well difference in the enzyme activity by the virus infection in every experiment. Ugai et al. (21) previously reported that the function of adenoviral vectors usually does not change unless they are exposed to excessive freeze/thaw cycles. 
We therefore assume that recombinant P450 activity in adenovirus-infected cells is not altered by limited cycles of freeze / thawing.

The fAd-CYP3A4 cells had higher CYP3A4 activity than Ad-CYP3A4 cells (Figure 2). The difference in activity levels among these cells corresponded to the mRNA and protein levels. This may be owing to the difference in total culture period after seeding the cells (fAd-CYP3A4 cells: 96 h, non-frozen Ad-CYP3A4 cells: 72 h). For the inhibition assays with ketoconazole, a potent inhibitor of CYP3A4-mediated metabolism, the $\mathrm{IC}_{50}$ values in fAd-CYP3A4 cells, Ad-CYP3A4 cells, and recombinant CYP3A4 microsomes were in good agreement with what has been reported $(20$, 22) (Figure 4 and Table 1). Moreover, hyperforin (one of SJW components) inhibited CYP3A4 activity and $\mathrm{IC}_{50}$ values were comparable on each experiment. Obach et al (23) previously reported that hyperforin potently inhibited of CYP3A4 activity in studies using recombinant CYP3A4 microsomes and hepatocytes (24). Moreover, Qi et al. (25) reported that oral clearance of midazolam in rats decreased with SJW treatment of short period. Therefore, our results suggest that fAd-CYP3A4 cells are a useful system to evaluate P450 inhibition and are comparable to human hepatocytes and microsomes.

Further, we have compared that inhibition effect to CYP3A4 enzymes activity by natural medicines on fAd-CYP3A4 cells and recombinant CYP3A4 microsomes. A previously published clinical study has shown that Cat's Claw interacts with HIV protease inhibitors, which are known CYP3A4 inhibitors $(26,27)$. Unger et al (28) reported that Devil's Claw, Feverfew, Peppermint Oil, and Red clover were inhibitors of CYP enzymes including CYP3A4. Although extent of inhibition of CYP3A4 activity by these natural medicines differed, all the natural medicine extracts exhibited this inhibitory effect (Figure 5). The inhibitory levels in recombinant CYP3A4 microsomes were stronger than those in case of fAd-CYP3A4 cells. In contrast, the CYP3A4 activity was strongly inhibited in fAd-CYP3A4 cells treated with Peppermint Oil extracts compared to that in the recombinant CYP3A4 microsomes. This difference might be involved in the permeability of the CYP3A4 inhibitors including these natural medicines into the cells. It was not possible to evaluate the cell membrane permeability of the compounds in microsomes unlike cell-based in vitro test systems for P450 inhibitors. In fact, Englund et al (29) have reported that $\mathrm{IC}_{50}$ values of elacrider for midazolam (CYP3A substrate) metabolism in hepatocytes were found to be higher than those found in liver microsomes. They have described that this difference may be due to the difference in membrane permeability of the inhibitor into the cells. Therefore, our results suggest that our evaluation system has reflected membrane permeability of compounds into the cells of livers. Our cell based-inhibitory assay system have mimicking human drug metabolism intracellular considered for membrane permeability.

Primary human hepatocytes are the most desirable enzyme source, but there are lot to lot ( donor to donor) variation and limitation to use the large amount of the each lot. Our results indicated that Ad-CYP3A4-infected cells could be cryopreserved and their activity did not decrease upon long-term storage. Thus, easy and reproducibly assessment is possible by storing a large volumes of fAd-CYP3A4 cells. Aoyama et al. (30) also have reported that HepG2 cells infected with two different varieties of P450-expressing adenovirus had no effect on each expression levels. Therefore, our model can be developed to evaluate a system that considers metabolic pathways in addition to other P450- and transporter-expressing adenoviruses.

In the present study, we have successfully developed a highly reproducible system, which provides large amounts of frozen CYP3A4-expressing cells after Ad-CYP3A4 infection. Our results suggested that this evaluation system become more similar to hepatocytes by expressing of multiple P450s and is more useful for screening inhibitory effect of natural medicine on P450 activity.

\section{ACKNOWLEDGMENTS}

This work was supported by a Health and Labour Sciences Research Grant for Research on Food Safety (No. 22230301) from the Ministry of Health, Labour and Welfare of Japan; and by the Japanese Ministry of Education, Culture, Sports, Science and Technology (MEXT)-Supported Program for the Strategic Research Foundation at Private Universities.

\section{REFERENCES}

1. Guengerich FP. Cytochrome P450s and other enzymes in drug metabolism and toxicity. AAPS J. 2006; 8(1):E101-11.

2. Guengerich FP. Cytochrome p450 and chemical 
toxicology. Chem Res Toxicol. 2008; 21(1):70-83.

3. Zanger UM, Schwab M. Cytochrome P450 enzymes in drug metabolism: regulation of gene expression, enzyme activities, and impact of genetic variation. Pharmacol Ther. 2013; 138(1):103-41.

4. Williams JA, Ring BJ, Cantrell VE, Jones DR, Eckstein J, Ruterbories $\mathrm{K}$, et al. Comparative metabolic capabilities of CYP3A4, CYP3A5, and CYP3A7. Drug Metab Dispos. 2002; 30(8):883-91.

5. Paine MF, Widmer WW, Hart HL, Pusek SN, Beavers KL, Criss AB, et al. A furanocoumarin-free grapefruit juice establishes furanocoumarins as the mediators of the grapefruit juice-felodipine interaction. Am J Clin Nutr. 2006; 83(5):1097-105.

6. Bailey DG, Malcolm J, Arnold O, Spence JD. Grapefruit juice-drug interactions. $\mathrm{Br} \quad \mathrm{J}$ Clin Pharmacol. 1998; 46(2):101-10.

7. Nowack R. Review article: cytochrome P450 enzyme, and transport protein mediated herb-drug interactions in renal transplant patients: grapefruit juice, St John's Wort - and beyond! Nephrology (Carlton). 2008; 13(4):337-47.

8. Piscitelli SC, Burstein AH, Chaitt D, Alfaro RM, Falloon J. Indinavir concentrations and St John's wort. Lancet. 2000; 355(9203):547-8.

9. Bauer S, Stormer E, Johne A, Kruger H, Budde K, Neumayer $\mathrm{HH}$, et al. Alterations in cyclosporin A pharmacokinetics and metabolism during treatment with St John's wort in renal transplant patients. Br J Clin Pharmacol. 2003; 55(2):203-11.

10. Brandon EF, Raap CD, Meijerman I, Beijnen JH, Schellens JH. An update on in vitro test methods in human hepatic drug biotransformation research: pros and cons. Toxicol Appl Pharmacol. 2003; 189(3):233-46.

11. Gomez-Lechon MJ, Donato MT, Castell JV, Jover R. Human hepatocytes as a tool for studying toxicity and drug metabolism. Curr Drug Metab. 2003; 4(4):292-312.

12. Vermeir M, Annaert P, Mamidi RN, Roymans D, Meuldermans W, Mannens G. Cell-based models to study hepatic drug metabolism and enzyme induction in humans. Expert Opin Drug Metab Toxicol. 2005; 1(1):75-90.

13. Donato MT, Lahoz A, Castell JV, Gomez-Lechon MJ. Cell lines: a tool for in vitro drug metabolism studies. Curr Drug Metab. 2008; 9(1):1-11.

14. Hewitt NJ, Hewitt P. Phase I and II enzyme characterization of two sources of HepG2 cell lines. Xenobiotica. 2004; 34(3):243-56.

15. Rodriguez-Antona C, Donato MT, Boobis A, Edwards RJ, Watts PS, Castell JV, et al. Cytochrome P450 expression in human hepatocytes and hepatoma cell lines: molecular mechanisms that determine lower expression in cultured cells. Xenobiotica. 2002; 32(6):505-20.

16. Gao X, Kim KS, Liu D. Nonviral gene delivery: what we know and what is next. AAPS J. 2007;
9(1):E92-104.

17. Ghosh SS, Gopinath P, Ramesh A. Adenoviral vectors: a promising tool for gene therapy. Appl Biochem Biotechnol. 2006; 133(1):9-29.

18. Sasaki T, Takahashi S, Numata Y, Narita M, Tanaka Y, Kumagai T, et al. Hepatocyte nuclear factor 6 activates the transcription of CYP3A4 in hepatocyte-like cells differentiated from human induced pluripotent stem cells. Drug Metab Pharmacokinet. 2013; 28(3):250-9.

19. Takezawa T, Matsunaga T, Aikawa K, Nakamura K, Ohmori S. Lower expression of HNF4alpha and PGC1alpha might impair rifampicin-mediated CYP3A4 induction under conditions where PXR is overexpressed in human fetal liver cells. Drug Metab Pharmacokinet. 2012; 27(4):430-8.

20. Greenblatt DJ, Zhao Y, Venkatakrishnan K, Duan SX, Harmatz JS, Parent SJ, et al. Mechanism of cytochrome P450-3A inhibition by ketoconazole. J Pharm Pharmacol. 2011; 63(2):214-21.

21. Ugai H, Watanabe S, Suzuki E, Tsutsui-Nakata H, Yokoyama KK, Murata T. Stability of a recombinant adenoviral vector: optimization of conditions for storage, transport and delivery. Jpn J Cancer Res. 2002; 93(5):598-603.

22. Qin CZ, Ren X, Tan ZR, Chen Y, Yin JY, Yu J, et al. A high-throughput inhibition screening of major human cytochrome P450 enzymes using an in vitro cocktail and liquid chromatography-tandem mass spectrometry. Biomed Chromatogr. 2014; 28(2):197-203.

23. Obach RS. Inhibition of human cytochrome P450 enzymes by constituents of St. John's Wort, an herbal preparation used in the treatment of depression. J Pharmacol Exp Ther. 2000; 294(1):88-95.

24. Komoroski BJ, Zhang S, Cai H, Hutzler JM, Frye R, Tracy TS, et al. Induction and inhibition of cytochromes $\mathrm{P} 450$ by the St. John's wort constituent hyperforin in human hepatocyte cultures. Drug Metab Dispos. 2004; 32(5):512-8.

25. Qi JW, Nakamura K, Hosokawa S, Okada Y, Horiuchi R, Yamamoto K. Time-dependent induction of midazolam-1-hydroxylation enzymes in rats treated with St. John's wort. Biol Pharm Bull. 2005; 28(8):1467-71.

26. Lopez Galera RM, Ribera Pascuet E, Esteban Mur JI, Montoro Ronsano JB, Juarez Gimenez JC. Interaction between cat's claw and protease inhibitors atazanavir, ritonavir and saquinavir. Eur J Clin Pharmacol. 2008; 64(12):1235-6.

27. Kumar S, Kumar A. Differential effects of ethanol on spectral binding and inhibition of cytochrome P450 3A4 with eight protease inhibitors antiretroviral drugs. Alcohol Clin Exp Res. 2011; 35(12):2121-7.

28. Unger M, Frank A. Simultaneous determination of the inhibitory potency of herbal extracts on the 
activity of six major cytochrome P450 enzymes using liquid chromatography/mass spectrometry and automated online extraction. Rapid Commun Mass Spectrom. 2004; 18(19):2273-81.

29. Englund G, Lundquist P, Skogastierna C, Johansson J, Hoogstraate J, Afzelius L, et al. Cytochrome p450 inhibitory properties of common efflux transporter inhibitors. Drug Metab Dispos. 2014; 42(3):441-7.

30. Aoyama K, Yoshinari K, Kim HJ, Nagata K, Yamazoe Y. Simultaneous expression of plural forms of human cytochrome $\mathrm{P} 450$ at desired ratios in HepG2 cells: adenovirus-mediated tool for cytochrome P450 reconstitution. Drug Metab Pharmacokinet. 2009; 24(3):209-17. 